\title{
Four Dimensional Scanning Transmission Electron Microscopy during the in situ Annealing of a CuZrAl Bulk Metallic Glass
}

\author{
Thomas C. Pekin ${ }^{1,2,3,}{ }^{*}$, Christoph Gammer ${ }^{4}$, Colin Ophus ${ }^{3}$, Robert O. Ritchie ${ }^{2,5}$, and Andrew M. Minor ${ }^{2,3}$ \\ 1. Department of Physics, Humboldt-Universität zu Berlin, Newtonstraße 15, 12489 Berlin, Germany. \\ 2. Department of Materials Science \& Engineering, University of California, Berkeley, CA 94720, USA. \\ 3. National Center for Electron Microscopy, Molecular Foundry, Lawrence Berkeley National \\ Laboratory, Berkeley, CA 94720, USA. \\ 4. Erich Schmid Institute of Materials Science, Jahnstraße 12, Leoben, Austria, 8700. \\ 5. Materials Sciences Division, Lawrence Berkeley National Laboratory, 1 Cyclotron Road, Berkeley, \\ CA 94720, USA. \\ *Corresponding author: tcpekin@physik.hu-berlin.de
}

Four dimensional scanning transmission electron microscopy (4D-STEM) is a powerful, diffraction-based imaging technique used to measure and map strain, polarization, and orientation, across a large field of view, typically in crystalline materials [1-5]. The technique relies on the processing and analysis of hundreds of thousands of rapidly acquired diffraction patterns to extract a rich set of information. 4DSTEM as a technique has been enabled by fast detectors and image processing codes capable of handling large amounts of data. The speed of data acquisition has in turn made in situ 4D-STEM possible, allowing for materials to be analyzed as they are dynamically perturbed $[4,6]$.

Recent developments in 4D-STEM analysis have been made in adapting this technique to amorphous materials $[6,7]$. The adaptation of 4D-STEM to amorphous materials has resulted in analysis that goes beyond fluctuation electron microscopy, which is the traditional electron microscopy based technique for amorphous specimens [8,9]. Particularly, the addition of a spatial component to the structural characterization of amorphous materials has allowed for the mapping of structural inhomogeneities that exist in bulk metallic glasses [7, 10]. Here, we extend 4D-STEM to in situ annealing of a bulk metallic glass $(\mathrm{BMG})$ to analyze the relationship between amorphous and crystalline phases during the crystalline phase's growth in a $\mathrm{Cu}_{46} \mathrm{Zr}_{46} \mathrm{Al}_{8} \mathrm{BMG}$.

Figure 1 shows the one frame from the from the in situ 4D-STEM time series. While the sample was annealed $400 \mathrm{C}$ for over two hours on a Protochips heating holder, this virtual bright field image shows the crystallite's size, from a region that originally had no crystallinity. From the same dataset, the virtual selected area diffraction patterns show the various crystalline orientations present in the crystallite, and their relationship to each other. These diffraction patterns can further be analyzed to show both amorphous and crystalline strain, simultaneously. Figure 2 shows subsequent ex situ energy dispersive x-ray spectroscopy (EDS) to provide chemical information of these phases. This research shows how 4D-STEM can be used to simultaneously study crystalline and amorphous phases in situ, to understand more fully the dynamic processes that govern phase nucleation and growth in amorphous materials [11].

\section{References:}

[1] V. B. Ozdol et al, Applied Physics Letters 106 (2015), p. 253107.

[2] D. Carvalho et al, Scientific Reports 6 (2016), p. 28459.

[3] E. Rauch and M. Véron, Materials Characterization 98 (2014), p. 1-9. 
[4] T. C. Pekin et al, Scripta Materialia 146 (2018), p. 87-90.

[5] K. Muller et al, Applied Physics Letters 101 (2012), p. 212110.

[6] C. Gammer et al, Applied Physics Letters 112 (2018), p. 171905.

[7] A. C. Y. Liu et al, Physical Review Letters 110 (2013), p. 205505

[8] P. M. Voyles, J. M. Gibson and M. M. J. Treacy, Journal of Electron Microscopy 49 (2000), p. 259266.

[9] P. M. Voyles and D. Muller, Ultramicroscopy 93 (2002), p. 147-159.

[10] S. Im et al, Ultramicroscopy 195 (2018), p. 189-93.

[11] The authors acknowledge support from the Director, Office of Science, Office of Basic Energy Sciences, Materials Sciences and Engineering Division, of the U.S. Department of Energy under Contract No. DE-AC02-05-CH11231 within the Mechanical Behavior of Materials program. Work at the Molecular Foundry was supported by the Office of Science, Office of Basic Energy Sciences, of the U.S. Department of Energy under Contract No. DE-AC02-05CH11231. T.C.P. acknowledges funding from the DFG project BR 5095/2-1 ("Compressed sensing in ptychography and transmission electron microscopy").
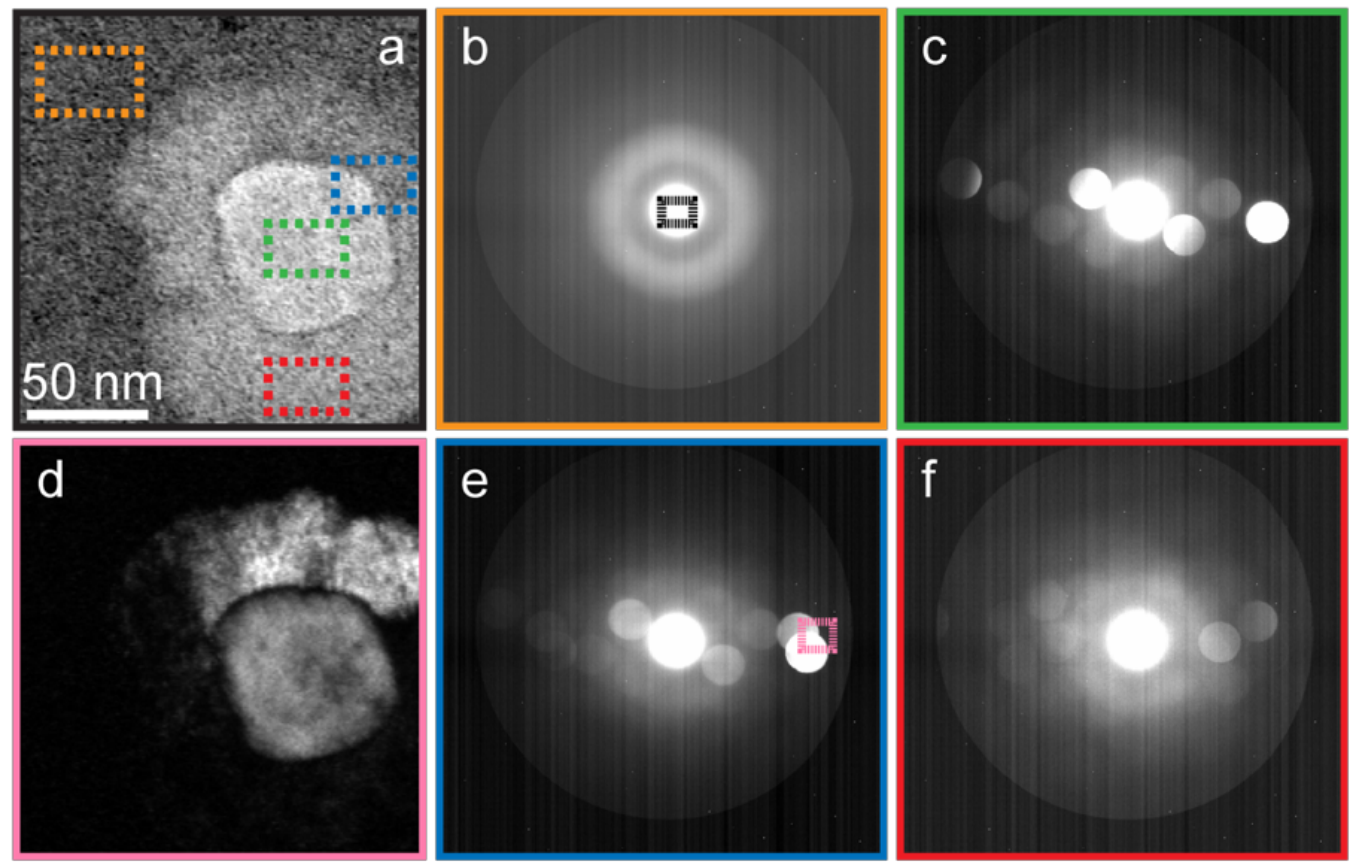

Figure 1. a) Virtual bright field image from the aperture shown in b), with color coded virtual selected area apertures. b,c,e,f) Virtual selected area diffraction patterns. d) Virtual dark field image from e), showing the orientation relationship between different crystalline phases.
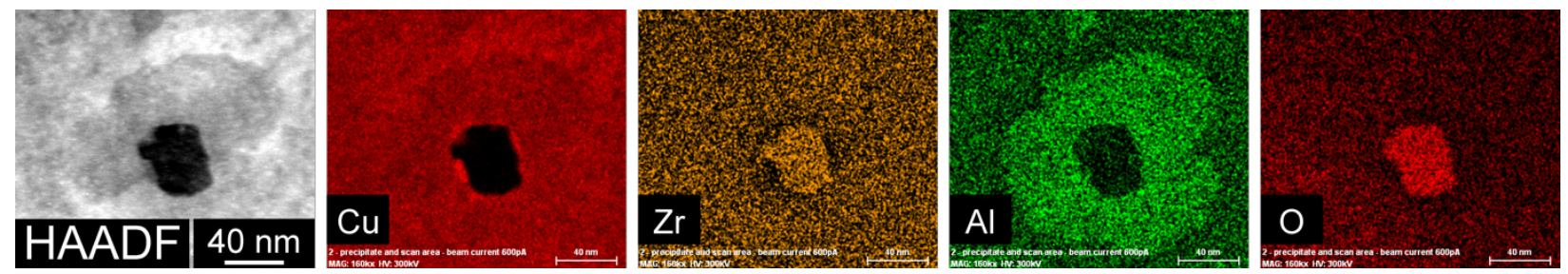

Figure 2. HAADF and EDS maps of crystalline and amorphous region from Fig. 1. Note the chemical segregation between the central $\mathrm{Zr}$ and O-rich region, and the Al-rich outer crystalline region. 